\title{
Impact of Used Communication Technology on the Navigation System for Hybrid Environment
}

\author{
Juraj Machaj, Peter Brida, and Michal Mlynka \\ Department of Telecommunications and Multimedia, University of Zilina, 01026 Zilina, Slovakia \\ Correspondence should be addressed to Juraj Machaj, machaj@fel.uniza.sk
}

Received 15 June 2012; Accepted 29 June 2012

Academic Editor: Stavros Kotsopoulos

Copyright ( 2012 Juraj Machaj et al. This is an open access article distributed under the Creative Commons Attribution License, which permits unrestricted use, distribution, and reproduction in any medium, provided the original work is properly cited.

This paper deals with navigation of mobile device in outdoor and indoor environment by only navigation system or application. In the paper, the navigation system is proposed in the light of seamless navigation service. Main parts of the system from positioning point of view are based on GPS and WifiLOC system. WifiLOC is an indoor positioning system based on Wi-Fi technology. The proposal of the system will be described in detail. The system is implemented at the University of Zilina as a pilot, noncommercial project; therefore it is called University Mobile Navigation System (UMNS). The navigation system can be characterized as realtime system, that is, the system operations cannot be significantly delayed. Since delay of the system depends significantly on communication platform used for map information downloading or communication with the localization server. We decided to investigate an impact of the used communication platform on the time needs for some of the functions implemented in navigation system. Measurements were performed in the real-world application. Next experiment is focused on testing of the accuracy of used indoor positioning system. Outdoor positioning accuracy is not tested because GPS is utilized in outdoor, and this system was already exhaustively investigated.

\section{Introduction}

In past few years, high number of navigation systems for mobile devices was successfully developed. Most of these systems can be used in outdoor environment, where position of the mobile device is estimated using satellite systems like GPS (Global Positioning System) or GLONASS (Global Navigation Satellite System). Generally, these systems are mainly used in transport applications, to find shortest path to the given destination point in the field of navigation, but can also be used for pedestrian navigation or navigation of the blind people [1-4].

Similar situation is in the indoor environment. Many navigation systems were developed also for this environment and they are mostly used for navigation in large buildings [5-7]. Most of these systems using radio signal positioning to achieve position estimate, which is important for the navigation applications [7]. It is also possible to use a highsensitivity GPS receivers or GPS pseudolites in the indoor environment, but this solution is still quite expensive $[8,9]$. The largest number of indoor navigation systems is based on the Wi-Fi technology and use fingerprinting positioning to estimate position of mobile device [10].

In this paper, we will propose navigation system for mobile device, which can be used in both indoor and outdoor environment. In the outdoor environment proposed system utilizes the GPS receiver, which is nowadays built in large number of smart phones. On the other hand, in the indoor environment positioning based on Wi-Fi fingerprinting localization is used to estimate position of the mobile device. In the navigation, position of the mobile device is estimated using WifiLOC positioning system [11]. This system has mobile-assisted centralised architecture, which means that measurements required for position estimation are performed by the mobile device. Measured data are then sent to the localization server, which estimates the position of mobile device.

Different communication technologies can be used by mobile device to connect with the servers, which are used to calculate optimal route to destination point, estimate position in the indoor environment or serve as storage of the maps. Since different technologies allow transmission 
with different data rates, impact of the used communication technology on the performance of proposed navigation system will be investigated.

Impact of the used communication technology on the communication time, together with localization accuracy in the indoor environment was investigated in real-world experiments. For this purpose, proposed navigation system was implemented at the campus of the University of Zilina.

Rest of the paper will be organized as follows; in the next section fingerprinting algorithm used for the indoor environment will be described. Proposed navigation system, described in detail in the Section 3. Section 4, introduces measurement scenarios. Achieved results are shown is Section 5. Section 6, concluding the paper and introducing plan for the future work.

\section{Fingerprinting Localization}

Fingerprinting algorithms can be divided into groups based on mathematical procedures used to estimate position of mobile device [12]. Basically, they can be divided into deterministic and probabilistic algorithms. In deterministic algorithms, the radio fingerprint is assumed to be nonrandom variable, which depends on the position of the device. On the other hand in probabilistic algorithms, the radio fingerprint is assumed as random variable. In our work we deal with deterministic fingerprinting algorithms based on nearest neighbor (NN) method, which can achieve sufficient accuracy as well as more complicated method, when density of radio map is high enough [13].

Accuracy of this method in radio networks is determined by two factors [14]. Firstly, signal properties vary much at relatively small area. For instance, in few meters range, signal from an AP can get attenuated, or even get lost. Secondly, these signals are relatively constant in time, what allows their use as indicators of a position.

Main disadvantage of this method is sensitivity to environment changes such as object movement in the building (e.g., people and furniture), which altogether affect signal properties. In case that environment has changed, it is necessary to update the map, but the signal is most affected by walls and furniture; therefore, update is not required so often.

2.1. Radio Map. Fingerprinting method consists of two phases. At first, it is creation of the radio map for an area where planned localization service is desired (see Figure 1). Radio map is basically a database of spots with known position (coordinates) coupled with various radio signal properties, for example, RSS, signal angles, or propagation time. The phase, when radio map is created, is called the offline phase. Generally, the offline phase can be performed by either measurements in a real environment or by prediction as described in [15]. In the first case, it is very timeconsuming, but there are precise real RSS information used in calculations. On the other hand, prediction of RSS is more comfortable, but the data are highly dependent on a quality of map model of given environment and also on used radio signal propagation model. High demands on

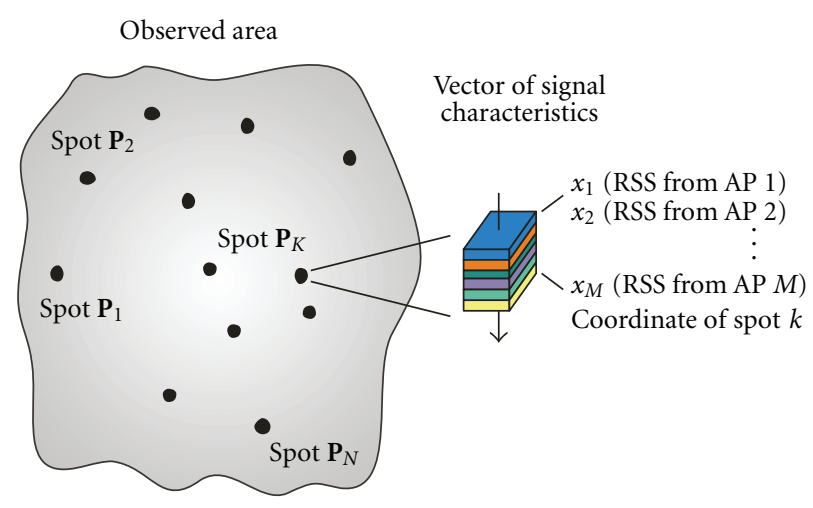

FIGURE 1: Radio map for fingerprinting algorithms using RSS.

the quality of map model and propagation model makes prediction of RSS quite hard to implement. Compromise between the demanding effort to create the radio map and achieved localization accuracy can be found in [16].

2.2. Localization Algorithm. After the radio map is created, second phase can take place. This phase is commonly called online or localization phase. During this phase MS measures signal properties at unknown spot. Then the radio map is searched to find a best match from existing spots. The match is actually the nearest point from database and is considered as MS position (for NN method).

The most common method how to find the best match and actually perform localization is use of the Euclidean distance with the Nearest Neighbor algorithm [12]. Let us assume a fingerprint in radio map, which is characterized by vector $\mathbf{P}$ :

$$
\mathbf{P}=\left[x_{j}\right]=\left[x_{1}, \ldots, x_{M}\right],
$$

where $x_{j}$ characterizes the spot, that is, values of the signal properties (e.g., RSS), $M$ represents the number of access points (APs) used for radio map creation. In general, let us consider the radio map contains fingerprints of $N$ spots:

$$
\mathbf{P}_{i}=\left[x_{i j}\right]=\left[x_{i 1}, \ldots, x_{i M}\right], \quad i=1, \ldots, N .
$$

Unique identifiers of neighbor APs as well as spot coordinates are stored in radio map and they are coupled with $x_{i}$, but are not shown here for model simplicity. The whole radio map contains all fingerprints $\mathbf{P}_{i}$ and creates the set $\mathbf{S}$ written as

$$
\mathbf{S}=\left\{\mathbf{P}_{i}: i=1, \ldots, N\right\} .
$$

In case of mobile device localization, the signal properties are measured at unknown spot, a new fingerprint $\mathbf{Q}$ is obtained as follows:

$$
\mathbf{Q}=\left[y_{j}\right], \quad j=1, \ldots, M .
$$

The Euclidean distance $d_{k}$ between vectors $\mathbf{P}_{k}$ and $\mathbf{Q}$ is defined as

$$
d_{k}=\left|\mathbf{P}_{k}-\mathbf{Q}\right|=\sqrt{\sum_{j=1}^{M}\left(x_{k j}-y_{j}\right)^{2}}
$$


When Euclidan distance formula is applied on the entire radio map, the vector of distances $\mathbf{D}$ is obtained between all of the radio map vectors $\mathbf{P}_{i}$ and the vector $\mathbf{Q}$ can be calculated as

$$
\mathbf{D}=\left[d_{i}\right]=\left[\left|\mathbf{P}_{i}-\mathbf{Q}\right|\right]=\left[\sqrt{\sum_{j=1}^{M}\left(x_{i j}-y_{j}\right)^{2}}\right], \quad i=1, \ldots, N .
$$

Position of mobile device than can be estimated using

$$
\hat{x}=\frac{\sum_{i=1}^{N}\left(1 / D_{i}\right) L_{i}}{\sum_{j=1}^{N}\left(1 / D_{j}\right)},
$$

where $\hat{x}$ is estimated position, $D_{i}\left(D_{j}\right)$ represents $i$ th $(j$ th) element of vector $\mathbf{D}, L_{i}$ stands for the position of $i$ th reference spot in the radio map database, and $N$ is the number of reference spots.

The estimator (7), which keeps $K$ positions with smallest distances is called the WKNNs (weighted $K$-nearest neighbors) method. WKNN with all distances $D_{i}=1$ is called the KNNs ( $K$-nearest neighbors) method [17]. The simplest method, where $K=1$, is called the NN (nearest neighbor) method.

\section{Proposal of Navigation System for Hybrid Environment}

We have developed navigation system feasible for navigation in both outdoor and indoor environments. The system is able to continuously navigate from outdoor to indoor environment or vice versa. Position used by the proposed navigation system can be estimated using GPS signals in outdoor environment, or using Wi-Fi localization system based on fingerprinting in the indoor environment and in areas, where the GPS signals cannot be used, due to NLOS (non-line of sight) conditions or high GPS signal attenuations.

The proposed navigation system was implemented at the University of Zilina as a pilot, noncommercial project; therefore, it is called University Mobile Navigation System (UMNS). This navigation system is developed as useroriented application for navigation in the hybrid environment using mobile device. Device should be equipped with Wi-Fi receiver, GPS receiver, internet connection and should be based on Android operation system. Basic concept of the proposed system is shown in Figure 2.

In the above figure is shown all equipments, which are used by the proposed navigation system to estimate position and estimate route to the given destination point. Communication between mobile device and servers is realized using different wireless technologies for the internet collection. Localization server is part of WifiLOC system and it is used to estimate position of mobile device in the indoor environment or in case that GPS signals are weak or not present. Indoor map server stores maps of the buildings, where navigation system can be used and it is represented by Ericsson Labs server. Outdoor map server is represented by Open MapQuest server, which stores outdoor maps of the world.
Geocode server is used to convert information provided by the user to geographic data. The server with OSM (Open Street Map) database stores information needed by both Open MapQuest and geocode servers.

Generally, if device is equipped with GPS receiver, and there is a visibility on the GPS satellites, the mobile device can estimate its position using directly GPS signals. On the other hand, when the mobile device is out of GPS signal range, for example, in indoor environment, position is estimated using the WifiLOC positioning system.

In case of the outdoor environment, map is downloaded from the Open MapQuest server. This open-source application is described in [18]. It is also possible to get information about actual geographic position, of the given address or add a name to the given position. In outdoor environment the route to the destination point is calculated on remote server, because of the large number of data. On the other hand, indoor maps are downloaded from the Ericsson labs servers [19]. At the beginning, the indoor maps have to be created on the server. We implemented this system as a case study of navigation service in the campus of University of Zilina; therefore, maps of the particular buildings of University of Zilina campus were created.

We decided to use Ericsson Labs servers because this server provides application to easily create maps of the indoor environment called Map Studio. Map Studio is accessible via internet connection as a web application. Advantage of this application is a possibility to create maps of any building, together with logic structure needed for the navigation. This tool also allows adding different points of interest and transfer points into the map. These points can used to compute optimal route for the navigation.

3.1. Principle of Communication in the System. In this part, the fundamental communication between parts of the system is explained in the following example. After the startup of the application in mobile device, the mobile device is localized on the map. The localization is automatically performed by GPS or WifiLOC. There is possibility to define destination point of the route if the user wants to be navigated. If user choose this option the map is shown together with menu as can be seen in Figure 3. From this menu the destination point for navigation can be chosen in three ways. First way is to choose the destination point directly from the map.

Second way is to search for the desired destination point. In that case user write desired destination as text request. Request should be written in format: street, city, and country. Request is then sent to geocode server. This server use text from the given request to find the position of the destination point using a data stored in the database. In case that request is not given in the specified format, geocode server can send back wrong coordinates of the destination point.

When the mobile device receives information from geocode server, position of destination is shown on the map using blue circle centred on the given position. In the case that geocode server does not find requested position, or there was an error in communication between device and geocode server, application shows message "Geocode server did not find requested position." 


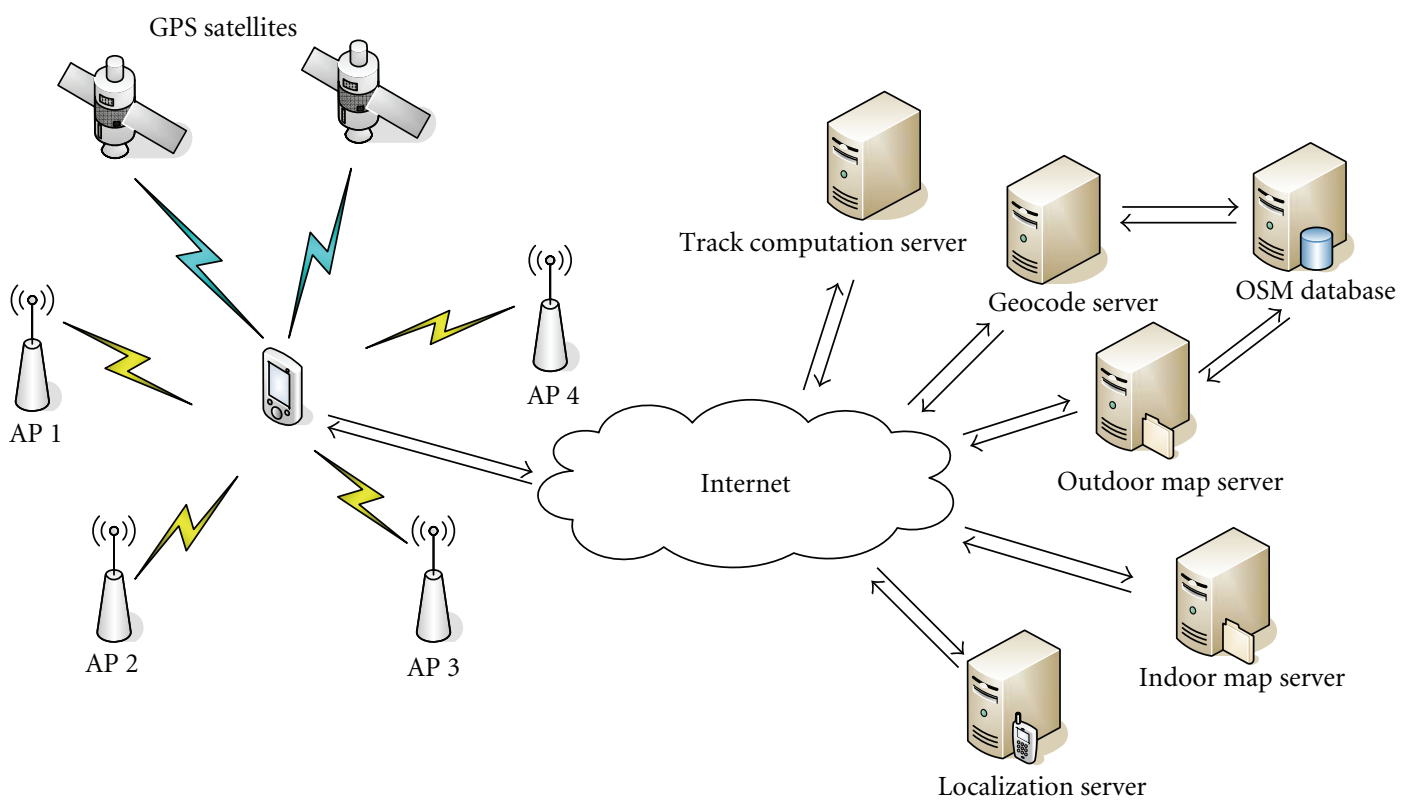

FIGURE 2: Concept of proposed navigation system.

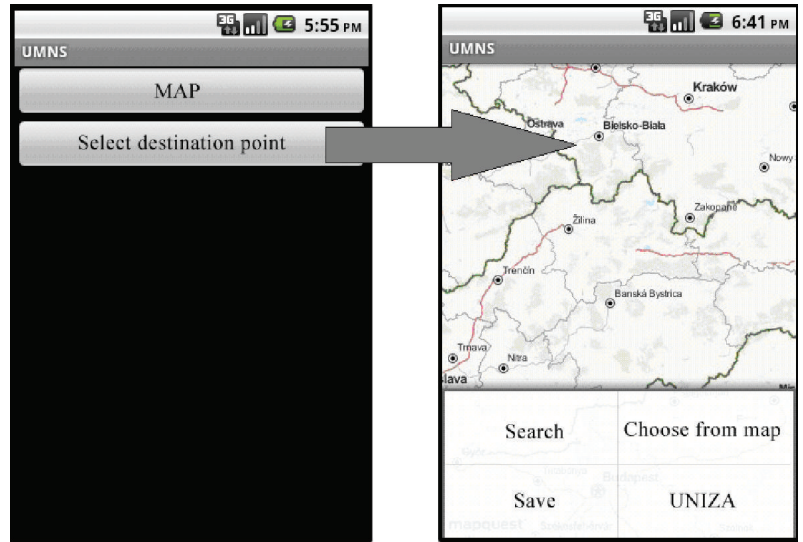

FIgURE 3: Menu to choose destination point of navigation.

Since UMNS allows also navigation inside the buildings, we implemented this system at the University of Zilina campus. There is button called UNIZA in the application menu. If the user chooses this option, it is possible to set the destination point inside the building of the university. It is possible to choose concrete building, floor, and so forth. According this information, the floor plan should be downloaded into the mobile device. This step is important because map data includes information about the whole building and also about every room. This information is needed to create a list of the rooms and a list of the persons, who have offices in the building. If data in the map was changed on the server, it is important to download the data again into the mobile device. When the maps are loaded, it is possible to choose destination point inside the building. Destination point can be chosen using room ID, or the name of a person which has office in the building from the list

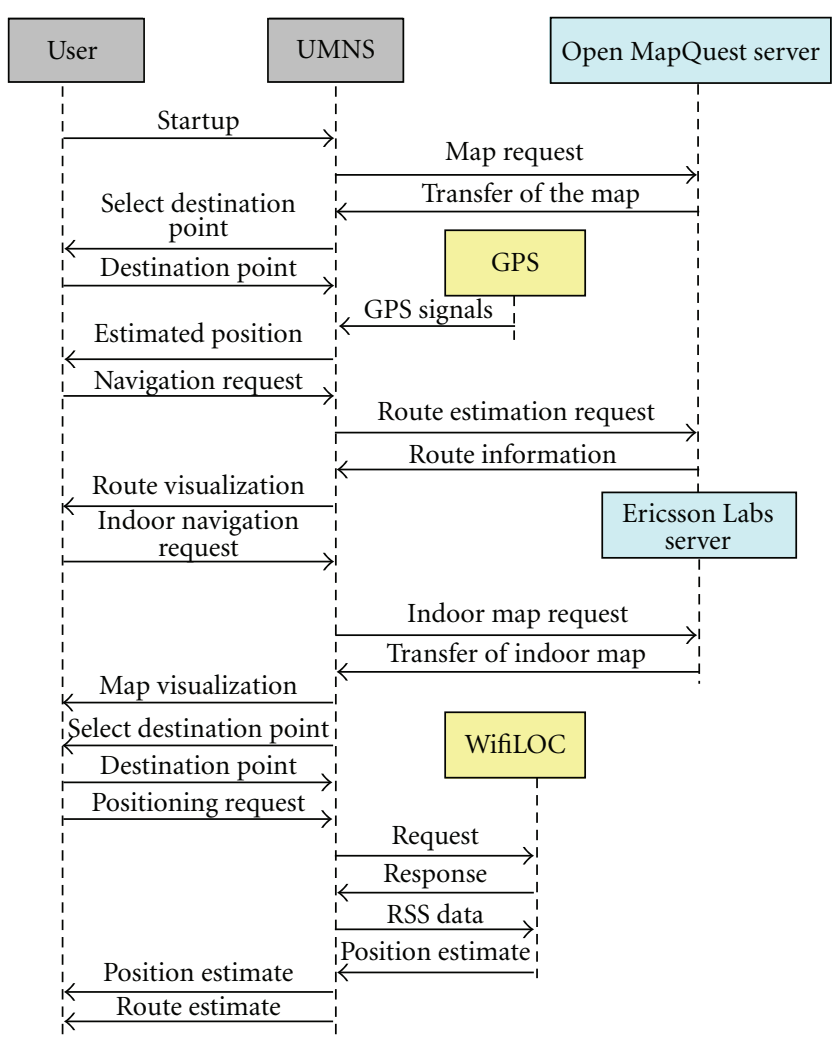

FIGURE 4: Sequence diagram of the communication in the proposed system.

generated by the application based on a data stored in the map. Sequence diagram of the communication between user, proposed UMNS application and servers is shown in the Figure 4. 
3.2. Navigation inside the Building. Maps downloaded from Ericsson Labs server are used during the navigation inside the building. In case that the user is navigated from the outdoor environment, his position is assumed to be at the building entrance. Application allows viewing the maps of the building. Each floor of the building has its own map and it is possible to switch between them by pressing point of interest (PoI) showing a stairs. In case of long press of the point in the map, information about room, like room ID and names of persons who have office in the given room, is shown.

Using settings menu it is possible to turn on localization of the mobile device or switch on or off the route to the destination point. Accuracy of the route is given by map properties and number of points in the map, since the route to destination point is calculated using points in the map and Dijkstra's algorithm. Localization in the indoor environment is implemented using the WifiLOC system or SelfLoc application. SelfLoc application is based on information given by the user of the mobile device. In SelfLoc, the user needs to define the point from which he wants to be navigated to the destination point. WifiLOC system was developed at the Department of Telecommunications and Multimedia at University of Zilina $[11,20]$. This localization system utilizes positioning based on Wi-Fi fingerprinting method.

3.3. WifiLOC Positioning System. The basic properties of the WifiLOC positioning system are described in this part. The system utilizes signal information from surrounding Wi-Fi networks for the position estimation. The system is based on the fingerprinting positioning method and received signal strength measurements.

WifiLOC system architecture, shown in the Figure 5, is designed as the mobile-assisted positioning concept. The mobile-assisted positioning means that the necessary measurements are done in the localized mobile device and measured results are forwarded to the localization server (LCS) in the network part. The position is estimated (calculated) at server side using basic NN algorithm (see Section 2).

The system is based on client-server architecture. From different point of view the entire architecture could be divided into three almost independent parts:

(i) localization server;

(ii) network of access points;

(iii) mobile device-user, client.

The division was purposely performed because of function of particular parts. The core component of the system is the localization server. It consists of more functional entities: database server, web server, and communication platform. LCS is built on Ubuntu operating system [21]. Radio map is saved on database server based on MySQL platform, which is free to use [22].

Communication between client and LCS could be implemented by various standard communication links depending on availability. Obviously, Wi-Fi is used as default, but bluetooth, UMTS (Universal Mobile Telecommunications System) or GSM (Global System for Mobile Communications) were successfully tested.
Network of access points can consist of various network provider APs. The signals from all fixed AP are passively scanned and utilized for the positioning. The system relies on the fact that transmission power is not changed when device is not connected to the AP.

Mobile device can be any mobile phone, personal device, tag or laptop equipped with IEEE 802.11 chipset. Localization application is developed in Java language SDK (Standard Development Kit) due to its easy implementation and crossplatform compatibility.

\section{Measurement Scenarios}

Navigation system described in previous chapter will be extensively tested for its parameters evaluation. Measurement scenarios were proposed to estimate the most important system parameters from navigation function point of view:

(a) time needed for the function of the proposed navigation system;

(b) indoor localization accuracy.

Time needed for the function of the navigation system is estimated as time from sending of the request till the end of the data processing on a mobile device. Each measurement was performed in the 50 independent trials. Sequence diagram for the measurements is shown in Figure 6. Concretely, there will be investigation into:

(a) time needed to download the map and route for indoor and outdoor navigation;

(b) time needed to download the map and navigation route (itinerary) for different route lengths in outdoor;

(c) time needed for position estimation by WifiLOC (indoor).

In the first scenario, measurements were performed to estimate time needed for maps downloading into the mobile device in outdoor environment. It is clear that downloading time is given by size of the given part of the map and also by used internet connection. The second part of this scenario was performed in the same way, but measurements were performed for downloading of indoor map instead of outdoor map. The impact of three different communication platforms was investigated: EDGE, UMTS/HSPA, and WiFi. We agreed on the platforms because they are the most widespread and implemented in almost all current mobile devices.

In the second scenario, time complexity of indoor localization part was estimated using time measurements similarly to the first scenario. In this case, estimated time was given by time needed to send the request for position estimation, time needed for position estimation by localization server, and time needed to send localization information from the localization server to the mobile device. Same communication technologies as in previous scenario were tested and compared. 


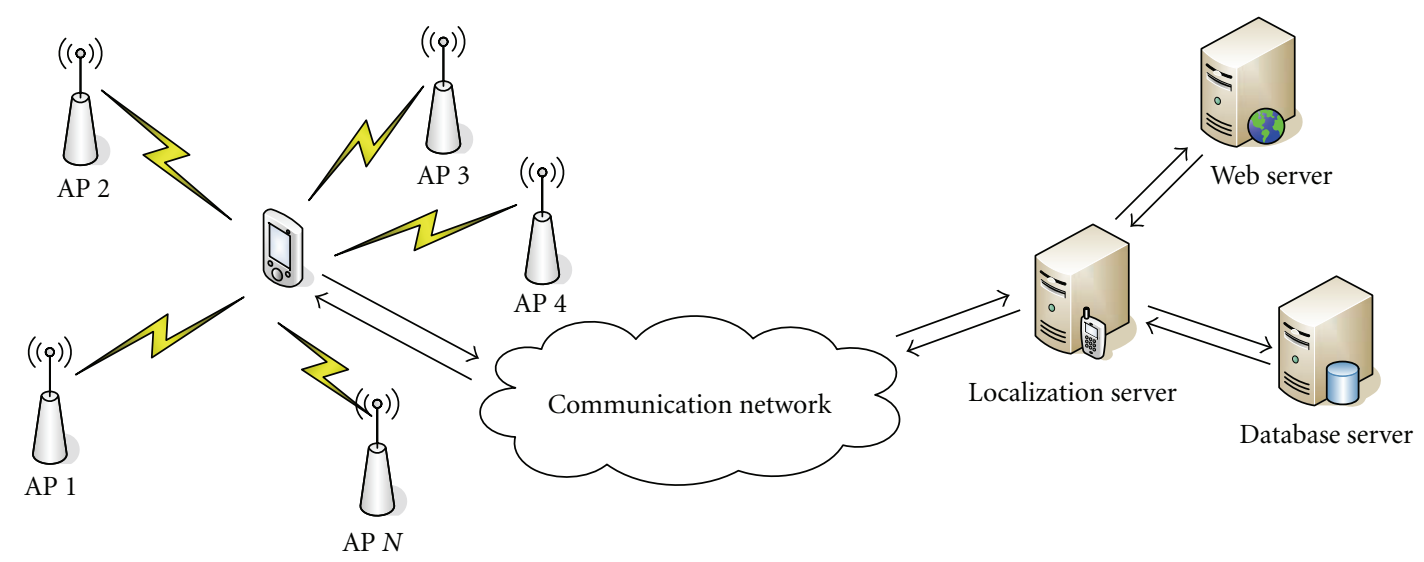

FIgURe 5: Architecture of the WifiLOC positioning system.

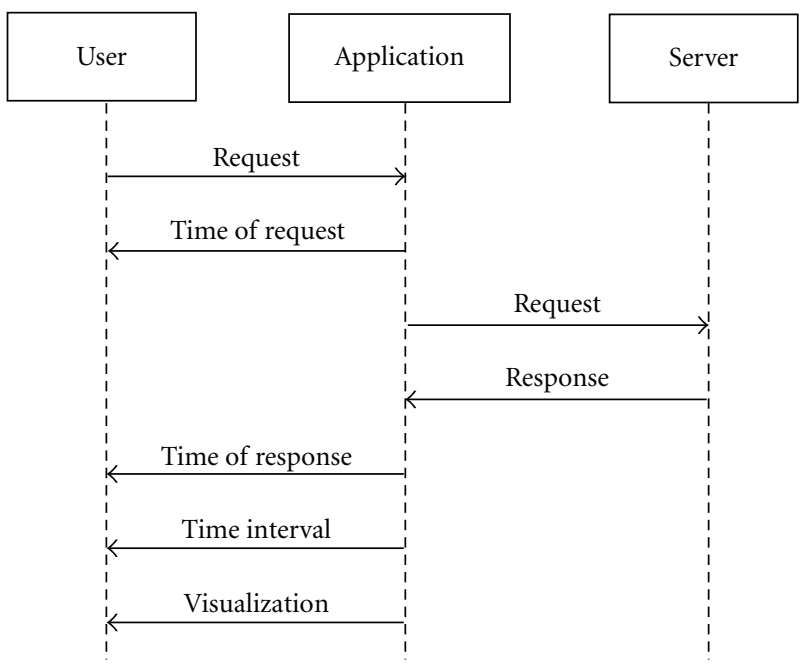

FIgURE 6: Sequence diagram for the time measurements.

In the third scenario, the accuracy of the WifiLOC positioning system was investigated. In this scenario, influence of the environment on the achieved localization accuracy was examined. Measurements were performed in different room types and during different times of the day. These measurements show how the small changes in environment caused by moving peoples affect achieved positioning error. In this scenario, position of mobile device was estimated in 50 independent trials for each of the four cases (200 position estimations in total). The area where localization accuracy of the WifiLOC system was tested is shown in the Figure 7. Localization area was situated on the one floor with size of approximately 333 meters squared. There were 67 spots in the radio map distributed in grid with regular distance of 2 meters.

Measurements performed in the last scenario were also aimed to evaluate positioning accuracy. In this scenario, measurements were performed during two days. This helps us to estimate impact of environment changes on the accuracy of proposed navigation system. Measurements can

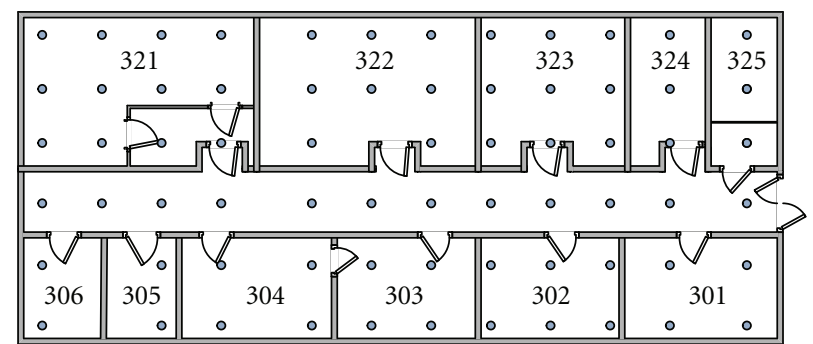

FIGURE 7: Environment where measurements were performed.

be divided into measurements performed in rooms and measurements performed in corridors. This helps us to investigate room accuracy of the localization algorithm. In this case, the measurements were performed for 80 positions in the rooms and 90 positions in the corridor during both days.

\section{Achieved Results}

In this section, results achieved during the measurements will be shown. All measurements were performed in the campus of the University of Zilina using HTC Legend mobile device. Results achieved in the first scenario are shown in Table 1.

According to the results shown in Table 1, there can be seen that the shortest time needed to map downloading was achieved by UMTS/HSPA communication platform. It can also be seen that mean time needed for the download of the map using Wi-Fi technology as communication platform, was almost the same compared to UMTS/HSPA. Disadvantage of Wi-Fi technology is high deviation of the time needed to download the map. This is probably given by background traffic in the Wi-Fi network generated by other users in the area. On the other hand, the advantage of communication over Wi-Fi platform lies in the economical point of view, since in most cases user is not charged any fees to use Wi-Fi network.

From the results it can also be seen that the longest time to download the map from the server was necessary for the EDGE communication platform. This result is not 
TABLE 1: Time needed to download the map and route for navigation.

\begin{tabular}{lcccc}
\hline & EDGE & UMTS/HSPA & Wi-Fi & \\
\hline$\Delta t(\mathrm{~ms})$ & 7891.03 & 1240.59 & 1383.22 & Outdoor \\
$\sigma(\mathrm{ms})$ & 2578.53 & 395.56 & 1495.28 & \\
$\Delta t(\mathrm{~ms})$ & 4456.52 & 1891.62 & 2135.72 & Indoor \\
$\sigma(\mathrm{ms})$ & 2628.99 & 437.74 & 651.62 & \\
\hline
\end{tabular}

TABle 2: Time needed to download the map and navigation route for different route lengths in outdoor environment.

\begin{tabular}{lcccc}
\hline & \multicolumn{3}{c}{ Used communication technology } & \multirow{2}{*}{ Length of the route } \\
& EDGE & UMTS/HSPA & Wi-Fi & \\
\hline$\Delta t(\mathrm{~ms})$ & 7891.09 & 1240.59 & 1383.22 & $d<10 \mathrm{~km}$ \\
$\sigma(\mathrm{ms})$ & 2578.53 & 395.56 & 1495.28 & \\
$\Delta t(\mathrm{~ms})$ & 12316.96 & 1551.57 & 1657.16 & $d<100 \mathrm{~km}$ \\
$\sigma(\mathrm{ms})$ & 6607.07 & 6607.07 & 423.39 & \\
$\Delta t(\mathrm{~ms})$ & 13722.92 & 2043.61 & 1871.86 & $d<500 \mathrm{~km}$ \\
$\sigma(\mathrm{ms})$ & 3886.06 & 353.09 & 738.38 & \\
$\Delta t(\mathrm{~ms})$ & 27242.92 & 2550.19 & 2992.82 & $d>500 \mathrm{~km}$ \\
$\sigma(\mathrm{ms})$ & 8668.71 & 829.79 & 445.13 & \\
\hline
\end{tabular}

surprising, due to much lower transfer speeds in the EDGE technology. It is commonly known that theoretical maximum transfer rate for EDGE technology is $384 \mathrm{kbps}$, UMTS/HSPA technology can achieve maximum transfer rate of $14 \mathrm{Mbps}$ for downlink and 5.74 Mbps for uplink and Wi-Fi technology can work with data transfer rates up to $54 \mathrm{Mbps}$.

When achieved results for the indoor and outdoor maps are compared, it can be seen that time needed to download maps has changed. Transfer time has decreased in the EDGE communication technology. On the other hand, particular times achieved for UMTS/HSPA and WiFi communication technologies increased. It was caused the fact that indoor maps are much smaller compared to outdoor maps. These results seem to be affected by speed of the login process needed for the connection to the server of Ericsson Laboratories, where the maps are stored.

In next step, time needed to download map and route for different lengths of the navigation route was measured. In this case, measurements were divided into four groups based on the length of the navigation route. First group was created for the route with distance smaller than $10 \mathrm{~km}$. Second group was created using distances in the range from $10 \mathrm{~km}$ to $100 \mathrm{~km}$. Third group consisted of distances in the rage between $100 \mathrm{~km}$ and $500 \mathrm{~km}$. The last and fourth group was created from routes with long distance navigations over $500 \mathrm{~km}$. Achieved results are shown in Table 2.

From the obtained results it can be seen that times achieved using UMTS/HSPA and Wi-Fi technologies were almost the same. It is also clear that difference between times needed to download the map and routes information when distance is lower than $10 \mathrm{~km}$ and $100 \mathrm{~km}$ are almost the same. Difference in the needed time is only $0.3 \mathrm{~s}$, it is very low. It can also be seen that time achieved using EDGE communication platform was much higher. In case
TABLE 3: Time needed for position estimation.

\begin{tabular}{lccc}
\hline & EDGE & UMTS/HSPA & Wi-Fi \\
\hline$\Delta t(\mathrm{~ms})$ & 1593.22 & 630.65 & 443.26 \\
$\sigma(\mathrm{ms})$ & 572.01 & 128.8 & 162.36 \\
\hline
\end{tabular}

that distance of the route is lower than $10 \mathrm{~km}$, time needed to download map and route information using EDGE is more than 5 times higher compared to Wi-Fi and UMTS/HSPA platforms. When distance to the destination is higher, results achieved using EDGE technology gets even worse and in case that map needed for long distance navigation needs to be downloaded the needed time is more than 9 times higher compared to Wi-Fi and UMTS/HSPA technologies.

Results achieved in the second scenario for delay of the position estimation, using WifiLOC localization system, are shown in Table 3. On the basis of the results it can be seen that communication technology seems to have significant impact on delay of estimated position.

It can be seen, that UMTS/HSPA and Wi-Fi communication technologies achieved almost the same delay of estimated position report. When communication between mobile device and localization server was based on connection via EDGE technology, delay of the estimated position was about $150 \%$ higher compared to Wi-Fi communication technology. From the results, it can be seen that time achieved using EDGE technology is too high for the navigation system application, since the time is higher than 1 second. Time of 1 second is assumed to be the highest position estimation delay time which is feasible for the navigation applications.

Results achieved in the third scenario can be seen in Figure 8. Measurements were performed in four cases, representing different conditions in the environment. In the first case both phases of the fingerprinting localization were performed during the same day, in the same conditions in the environment. In the case number 2 , the localization phase was performed during the other day, there was different number of moving people in the area and few pieces of furniture were moved. In the third case, the online phase was performed in the empty building, so there were no moving people that can affect the signal propagation. All the measurements performed in the fourth case were done when there were no moving people in the building during the both phases.

From the results it can be seen that changes in the environment have impact on localization accuracy. It can be seen that, when there was no moving people during the localization phase, accuracy achieved was much lower even if the radio map was created in different environment conditions (case 3). It is clear that best results were achieved in case 4 representing ideal conditions, because disturbances caused by the dynamic changes of the environment were minimized. On the other hand, the worst results were achieved in case 2 which can be identified as real conditions since it represents most common environment in real-world applications. 
TABLE 4: Localization precision of WifiLOC positioning system.

\begin{tabular}{|c|c|c|c|c|c|}
\hline & & Number of estimations & Number of accurate estimations & Number of false estimations & Success rate $(\%)$ \\
\hline \multirow{2}{*}{ Rooms } & Day 1 & 80 & 74 & 6 & 92.50 \\
\hline & Day 2 & 80 & 71 & 9 & 88.75 \\
\hline \multirow{2}{*}{ Corridor } & Day 1 & 90 & 76 & 14 & 84.44 \\
\hline & Day 2 & 90 & 77 & 13 & 85.56 \\
\hline
\end{tabular}

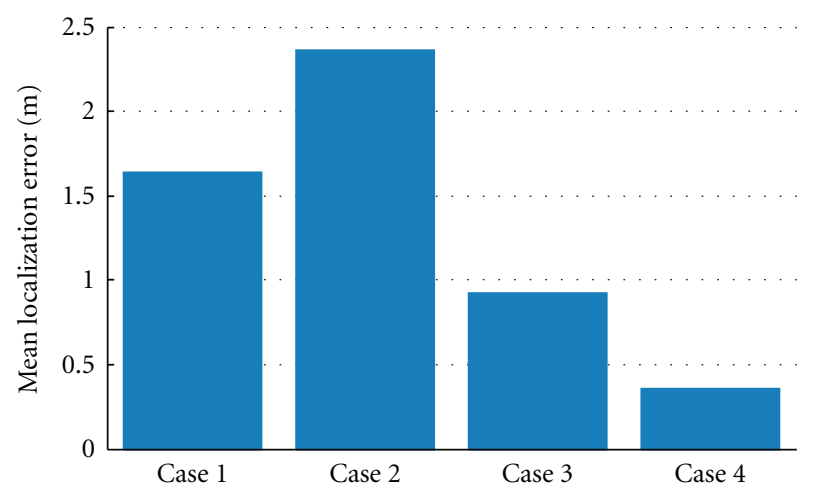

FIGURE 8: Localization error achieved in the different conditions in the indoor environment.

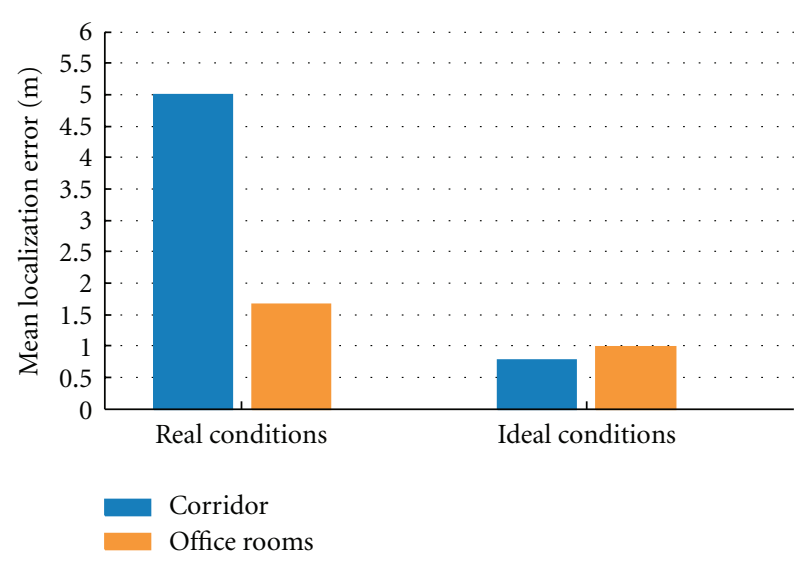

FIgURE 9: Localization error achieved in the different conditions and different types of the indoor environment.

In the next step, localization accuracy achieved for both ideal and real conditions, for two different types of rooms was compared. Rooms were divided into office rooms and corridors, achieved results can be seen in Figure 9.

According to achieved results it can be seen that localization error depends on the type of room in the environment. In the real conditions, localization accuracy in the office rooms was much higher compared to corridor. This is caused by differences in propagation and by different impact of the moving people on the localization accuracy. For the office rooms, the difference in achieved localization error is much lower compared to change in corridor.

In the last scenario, measurements were performed to evaluate positioning precision of the proposed navigation system. In this case, measurements were done in the real conditions, in different days and in all kinds of above investigated environment. Localization precision is evaluated by comparison of estimated and real positions. When estimated position is in the same area (same office room or same part of the corridor), then estimated position is marked as accurate, and in case that estimated position is not in the same area position is marked as false estimation. Achieved results are shown in Table 4.

From the table, it can be seen that higher precision was achieved in rooms compared to corridors. It can be seen that in case that mobile device was in the room, positioning precision was about $90 \%$. It is also evident that number of false estimated positions was almost the same during both days, so precision of the positioning system seems to be stable even in case that there are some minor changes in the localization area.

In the corridor, number of false estimated positions was slightly higher; accurate estimations achieved $5 \%$ worse value compared to results achieved in rooms. This can be caused by the fact that Wi-Fi signals in corridors have a bit different characteristics due to differences in propagation of radio waves. The differences in the accuracy in the corridors, when there are minor changes in the environment, are even smaller compared to results achieved in the rooms.

Results achieved in the measurements show that localization accuracy of the used localization system for indoor positioning should be improved. Results also show that there is impact of the used communication platform on the delay of the estimated position report. The worst results were achieved by EDGE communication technology, but even delay achieved by EDGE technology is suitable for the proposed application.

\section{Conclusion and Future Works}

In this paper, a novel navigation system applicable to the hybrid environment was described. The system can provide a navigation service in the hybrid environment where mobile device has to be localised in outdoor in indoor. The system was implemented as University Mobile Navigation System (UMNS) at the campus of the University of Zilina. The real-world testing of the system was performed in this environment.

Real-world measurements were performed to evaluate time complexity of localization and map and route downloading using different communication platforms. According to the achieved, results we can conclude that communication platform has significant impact on the time necessary for a relevant data downloading. As we expected, the most reliable platform was UMTS/HSPA from a time relations point of 
view. The best results were achieved by the UMTS/HSPA platform for both outdoor and indoor environments. Communication based on the Wi-Fi technology achieved similar results compared to UMTS/HSPA from the time delay point of view. Advantage of Wi-Fi technology lies in the fact, that communication via this technology is mostly free of charge. Communication based on EDGE technology had the highest delay. These results are caused by the transport speeds of particular communication technologies.

Delay of the position estimation was only investigated in the indoor environment, because GPS was used in outdoor environment and it is a well-known tested system. Indoor positioning was performed by part of the system based on WifiLOC positioning system. Achieved results have almost the same characteristic as previous results for all used communication platforms. Delay of the position estimation achieved using Wi-Fi and UMTS/HSPA was almost the same. When EDGE platform was used for communication with localization server, achieved delay was much higher. From the achieved results, it is clear that EDGE communication platform is not feasible for the navigation purposes due to high delay of the position estimation.

In the last experiment, the positioning accuracy in indoor was extensively investigated. The changes of the localization area during both offline and online phases have significant impact on positioning accuracy. Therefore, the radio map should be periodically optimized. Finally, the successful position estimation rate was tested. The position estimation was successful if the estimated position is in the true area (same office room or same part of the corridor). The successful position estimation rate was more than $85 \%$ of all position estimations. These results confirm that idea about positioning seems to be optimal for positioning and navigation in hybrid environment. The accuracy of the used indoor positioning system should be increased. The success rate can be improved by implementation of optimization algorithm which takes into account previous position of the mobile device. The implementation of the optimization algorithm to the navigation system belongs to our future research goals.

\section{Acknowledgments}

This work has been partially supported by the Slovak Research and Development Agency under Contract no. LPP0126-09 and by Centre of excellence for systems and services of intelligent transport II., ITMS 26220120050 supported by the Research \& Development Operational Programme funded by the ERDF.

\section{References}

[1] S. Jung and W. Woo, "UbiTrack: infrared-based user Tracking System for indoor environment," in Proceedings of the International Conferece on Artificial Reality and Telexisitence (ICAT '04), pp. 181-184, Coex, Korea, 2004.

[2] H. Makino, I. Ishii, and M. Nakashizuka, "Development of navigation system for the blind using GPS and mobile phone combination, engineering in medicine and biology society," in Proceedings of the 18th Annual International Conference of the
IEEE Engineering in Medicine and Biology Society, vol. 2, pp. 506-507, November 1996.

[3] M. Hao and S. Xi, "Research of TETRA terminal navigation and location system based on GPS/GIS," Instrument Standardization and Metrology, no. 5, pp. 18-19, 2006.

[4] G. Hong-wei, B. Xiang, and Q. I. Qin, "On positioning and navigation of mobile-phone based on gpsOne," Microcomputer Application Technology, no. 69, pp. 34-37, 2007.

[5] O. Cruz, E. Ramos, and M. Ramirez, "3D indoor location and navigation system based on Bluetooth," in Proceedings of the 21st International Conference on Electronics Communications and Computers (CONIELECOMP '11), pp. 271-277, March 2011.

[6] S. Chumkamon, P. Tuvaphanthaphiphat, and P. Keeratiwintakorn, "A blind navigation system using RFID for indoor environments," in Proceedings of the 5th International Conference on Electrical Engineering/Electronics, Computer, Telecommunications and Information Technology (ECTI-CON '08), pp. 765-768, tha, May 2008.

[7] Y. Jiang, Y. Fang, C. Yao, and Z. Wang, "A design of indoor \& outdoor navigation system," in Proceedings of the IET International Conference on Communication Technology and Application (ICCTA '11), pp. 877-881, 2011.

[8] S. Schön and O. Bielenberg, "On the capability of high sensitivity GPS for precise indoor positioning," in Proceedings of the 5th Workshop on Positioning, Navigation and Communication (WPNC '08), pp. 121-127, Hannover, Germany, March 2008.

[9] A. Vervisch-Picois and N. Samama, "Interference mitigation in a repeater and pseudolite indoor positioning system," IEEE Journal of Selected Topics in Signal Processing, vol. 3, no. 5, pp. 810-820, 1932.

[10] B. Dawes and K.-W. Chin, "A comparison of deterministic and probabilistic methods for indoor localization," Journal of Systems and Software, vol. 84, no. 3, pp. 442-451, 2011.

[11] P. Brida, F. Gaborik, J. Duha, and J. Machaj, "Indoor positioning system designed for user adaptive systems," in Proceedings of the 3rd Asian Conference on Intelligent Information and Database Systems (ACIIDS '11), pp. 237-245, Daegu, South Korea.

[12] H. Laitinen, J. Lähteenmäki, and T. Nordström, "Database correlation method for GSM location," in Proceedings of the IEEE VTS 53rd Vehicular Technology Conference (VTS SPRING '01), pp. 2504-2508, May 2001.

[13] L. Tsung-Nan and L. Po-Chiang, "Performance comparison of indoor positioning techniques based on location fingerprinting in wireless networks," in Proceedings of the International Conference on Wireless Networks, Communications and Mobile Computing, vol. 2, pp. 1569-1574, June 2005.

[14] V. Otsason, Accurate indoor localization using wide GSM fingerprinting [M.S. thesis], Tartu, Estonia, 2005.

[15] T. Chrysikos, G. Georgopoulos, K. Birkos, and S. Kotsopoulos, "Wireless channel characterization: on the validation issues of indoor RF models at $2.4 \mathrm{GHz}$," in Proceedings of the 1st Panhellenic Conference on Electronics and Telecommunications (PACET '09), Patras, Greece, March 2009.

[16] K. R. Anne, K. Kyamakya, F. Erbas, C. Takenga, and J. C. Chedjou, "GSM RSSI-based positioning using extended Kalman filter for training artificial neural networks," in Proceedings of the IEEE 60th Vehicular Technology Conference: Wireless Technologies for Global Security (VTC-Fall '04), pp. 4141-4145, Los Angeles, Calif, USA, September 2004.

[17] V. Honkavirta, T. Perälä, S. Ali-Löytty, and R. Piché, "A comparative survey of WLAN location fingerprinting methods," in 
Proceedings of the 6th Workshop on Positioning, Navigation and Communication (WPNC '09), pp. 243-251, March 2009.

[18] OpenStreetMap. MapQuest, OpenStreetMap Community, 2011, http://wiki.openstreetmap.org/wiki/MapQuest.

[19] Ericsson, Ericsson Labs, Ericsson Labs-Indoor Maps, 2011, http://indoor.labs.ericsson.net/.

[20] P. Brida, J. Benikovsky, and J. Machaj, "Performance investigation of WifiLOC positioning system," in Proceedings of the 34th International Conference on Telecommunications and Signal Processing (TSP '11), pp. 203-207, Budapest, Hungary, 2011.

[21] Canonical Ltd. Ubuntu, http://www.ubuntu.com/.

[22] MySQL, http://www.mysql.com/. 

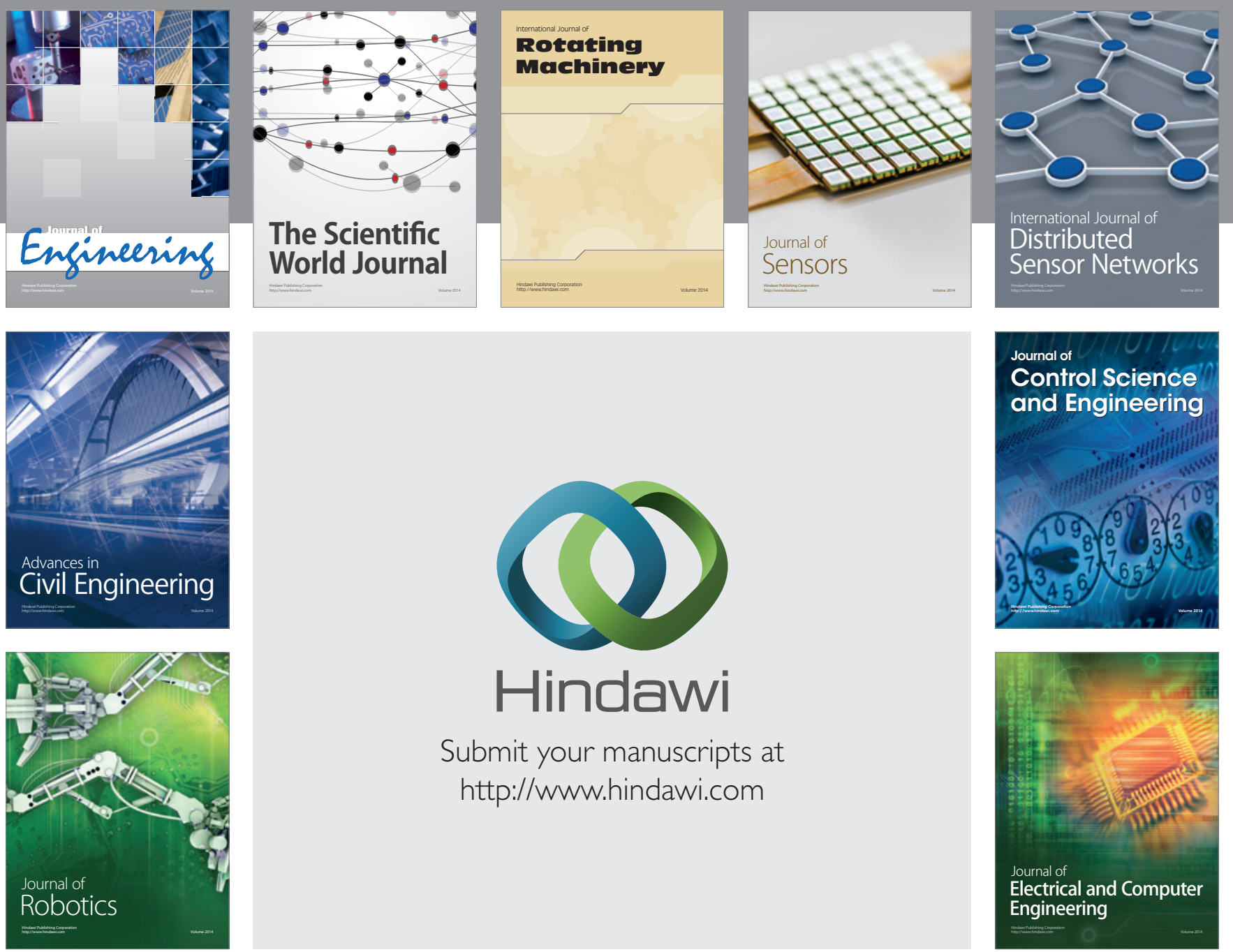

Submit your manuscripts at

http://www.hindawi.com
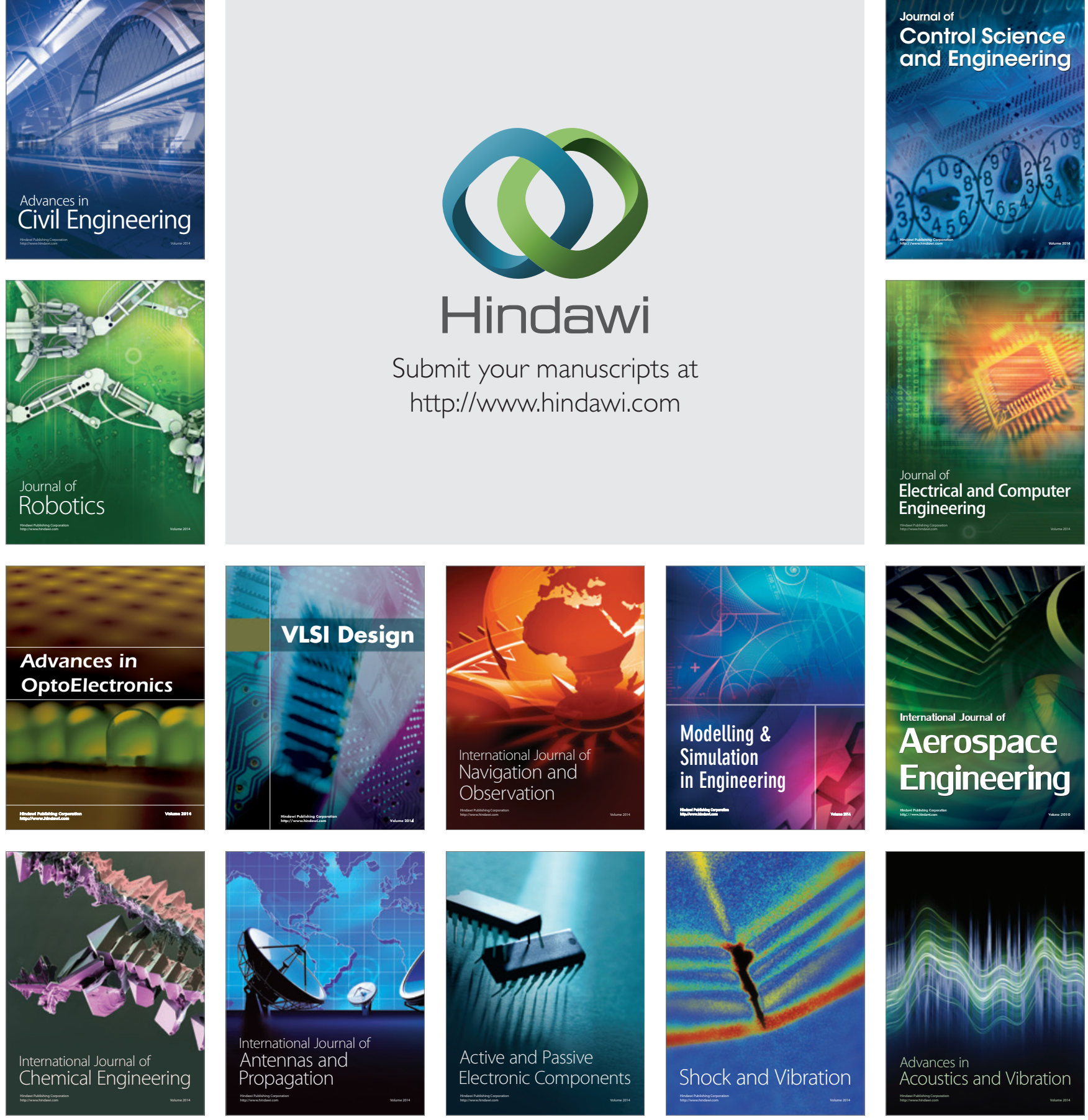